\title{
Have food portion sizes increased in the UK over the last 20 years?
}

\author{
W. Wrieden, A. Gregor and K. Barton \\ University of Dundee, Dundee, $U K$
}

Current published food portion sizes were estimated from data collected in the 1986-7 Dietary and Nutritional Survey of British Adults. However, there is evidence from the USA that portion sizes for specific foods, e.g. soft drinks, hamburgers and French fries, have increased over the last 20 years $^{(1,2)}$.

Using the latest data available from the National Diet and Nutrition Surveys (NDNS) of adults aged 19-64 years, median portion weights were calculated ${ }^{(3)}$ and compared with those estimated from 1987 as average or medium portions in Food Portion Sizes ${ }^{(4)}$ and as the median portion deduced from the data given in A Photographic Atlas of Food Portion Sizes ${ }^{(5)}$. In addition, a range of companies including food manufacturers, supermarkets and fast-food and restaurant chains were contacted and information on portion sizes obtained and compared with those collected in the 1990s.

Of the fifty-seven average food portion sizes that could be directly compared, only six, all savoury items, showed an increased median size $>5 \%$ of those reported earlier. In contrast, portion sizes had decreased or stayed the same for five types of potatoes or chips, nineteen fruits and vegetables and twelve dessert items.

\begin{tabular}{|c|c|c|c|c|c|c|c|}
\hline \multirow[b]{2}{*}{ Food } & \multicolumn{3}{|c|}{ Food portion sizes* } & \multicolumn{2}{|c|}{ Photographic atlas* } & \multicolumn{2}{|c|}{ NDNS 19-64 years (2000)* } \\
\hline & $\mathrm{S}$ & $\mathrm{M} / \mathrm{Av}$ & $\mathrm{L}$ & Median & IQR & Median & IQR \\
\hline Macaroni cheese & & 220 & & 195 & $111-280$ & 281 & $220-365$ \\
\hline Porridge & 110 & 160 & 210 & 204 & $108-299$ & 220 & $175-271$ \\
\hline Stewed mince & & 140 & 220 & 166 & $91-240$ & 181 & $130-242$ \\
\hline $\begin{array}{l}\text { Chilli con carne/bolognaise with rice } \\
\text { or spaghetti }\end{array}$ & & 290 & & 274 & $177-370$ & 350 & $300-400$ \\
\hline Cod or haddock fried in batter & 120 & 170 & 220 & 166 & $108-218$ & 180 & $162-200$ \\
\hline Soup & 150 & 220 & 300 & 229 & $120-338$ & 271 & $200-363$ \\
\hline
\end{tabular}

$\mathrm{S}$, small; M/Av, medium or average; L, large; IQR, interquartile range. *Values expressed in $\mathrm{g}$.

Examination of portion sizes of manufactured confectionery items identified seventy-one items that could be directly compared, of which twelve had decreased and twelve had increased in size. However, a greater range of items appeared to be available with the introduction of king and giant sizes. Increased portion sizes were seen in two international fast-food chains; twelve of twenty-six comparable items had increased in portion size and only two had decreased. The increases were seen in hamburgers (equivalent to an increase of 50-213 kJ) and chicken items, but not French fries, although one chain has introduced a super portion.

In summary, there is some evidence of increasing portion sizes in fast-food outlets in the UK, but the increases in energy intakes resulting from this change are not as large as those reported in the USA. This difference may reflect the longer time period measured in the USA, for which comparison was with data from the 1970s to the mid 1990s. Nevertheless, the fact that increases were seen in the fastfood items from international fast-food outlets is a cause for concern given the increasing levels of obesity.

Funding provided by Food Standards Agency is gratefully acknowledged (Project no. N08018).

1. Young LR \& Nestle M (2002) Am J Public Health 92, 246-249.

2. Nielsen SJ \& Popkin BM (2003) JAMA 289, 450-453.

3. Barton KL, Wrieden WL, Adamson AJ \& Cochrane L (2006) Proc Nut Soc 65, 97A

4. Food Standards Agency (2002), Food Portion Sizes, 3rd ed. London: H M Stationery Office.

5. Nelson M, Atkinson M \& Meyer J (1997) A Photographic Atlas of Food Portion Sizes, London: MAFF Publications. 